\title{
An imaging of buried anomalies, using multi-sheet inversion
}

\author{
Dmitry B. Avdeev, Alexei V. Kuvshinov, and Oleg V. Pankratov \\ Institute of Geoelectromagnetic Research, Russian Academy of Sciences, 142092 Troitsk, Moscow Region, Russia
}

(Received January 26, 1996; Revised November 17, 1997; Accepted February 17, 1998)

\begin{abstract}
The objective of this paper is to construct a stable inversion scheme to determine the conductance of a buried inhomogeneous layer masked by sedimentary cover. We employ a model which incorporates a surface sheet of conductance $S_{1}(x, y)$ and a buried sheet of conductance $S_{2}(x, y)$. The buried sheet is embedded at a depth $d$ in a layered earth of conductivity $\sigma(z)$. The $\sigma(z), d$ and $S_{1}(x, y)$ are assumed to be known. We determine $S_{2}(x, y)$ by inverting the monochromatic horizontal electromagnetic (EM) field measured at the earth's surface. To do this, we use Price's matching conditions at the sheets and a stable continuation of EM field from the earth's surface onto the buried sheet. Employing synthetic data we demonstrate that the designed inversion scheme generates a reasonable image of the buried structure. The scheme is fast, since it doesn't require any forward modelling.
\end{abstract}

\section{Introduction}

One of the most challenging problems in geoelectrics is developing reliable and stable approaches of imaging inhomogeneous underground structures using electromagnetic (EM) data. Obviously, a rigorous inversion within fully threedimensional (3-D) earth's models (Eaton, 1989; Madden and Mackie, 1989; Oldenburg and Ellis, 1991; Lee and Xie, 1993; Alumbaugh, 1993; Newman, 1995) is best suited for adequate imaging. However the rigorous inversion stands in need of repeated 3-D forward modelling and so it appears to be very time-consuming procedure. To reduce computational loads quasi 3-D earth's models are often considered. One of the useful simplified 3-D models being exploited for both modelling and inversion is a multi-sheet model (Singer and Fainberg, 1985).

In this paper, using the multi-sheet model, we develop a novel approach of imaging conductors underlain by a sedimentary layer of known conductance. The model is composed of a surface and a buried conducting sheet embedded in a layered earth (Fig. 1). Our approach is conceptually close to that by Avdeev et al. (1986) however it exploits a new, stable (but still adequate) approximation of a downward continuation of the surface EM field.

To test our imaging approach, synthetic EM data are inverted. Two kinds of excitation were considered: a grounded electric dipole and a plane wave. We show that a trustworthy image of the buried sheet is recovered for both kinds of excitation.

\section{Inverse Problem Formulation}

Let's consider the multi-sheet model to be used (Fig. 1). The model consists of a surface sheet of conductance $S_{1}(x, y)$ and a buried sheet of conductance $S_{2}(x, y)$. The buried sheet is embedded at a depth $d$ in a layered earth of conductivity $\sigma(z)$. The surface sheet contacts with insulating half-space

Copy right (C) The Society of Geomagnetism and Earth, Planetary and Space Sciences (SGEPSS); The Seismological Society of Japan; The Volcanological Society of Japan; The Geodetic Society of Japan; The Japanese Society for Planetary Sciences. $z \leq 0$ (air). The magnetic permeability is assumed to be $\mu_{0}$ everywhere. The model is excited by a monochromatic current $\mathbf{j}^{\text {ext }}(x, y, z ; \omega)$ of frequency $\omega$. The time harmonic dependence is $\exp (-i \omega t)$, where $i=\sqrt{-1}$. We now consider the inverse problem formulation. We suppose the following things as known: horizontal electric $\mathbf{E}_{\tau}(x, y, 0, \omega)$ and magnetic $\mathbf{H}_{\tau}(x, y, 0, \omega)$ fields at the earth's surface $(z=0)$; the conductivity $\sigma(z)$; the conductance $S_{1}(x, y)$; and the depth $d$. The problem is then to find the conductance $S_{2}(x, y)$, which is an inverse problem. A limitation of this inverse problem formulation is related to the fact that adequate spatial coverage and simultaneous EM measurements are required. Another limitation is that the frequency $\omega$ must be small enough that approximation of inhomogeneous structures by thin sheets is valid. It means that we assume that the electric field $\mathbf{E}_{\tau}$ varies only negligably across both surface and buried sheets (Price, 1949).

\section{Basic Concepts of the Inverse Problem Solution 3.1 Determination of buried sheet conductance}

In this subsection we assume for the moment that the electric $\mathbf{E}_{\tau}(x, y, d, \omega)$ and magnetic $\mathbf{H}_{\tau}(x, y, d-0, \omega)$ fields at the roof of the buried sheet $(z=d-0)$ are known. Then $S_{2}(x, y)$ can be found as follows (Avdeev et al., 1986, 1994). Given $\mathbf{E}_{\tau}(x, y, d)$ and $\sigma(z)$, we determine $\mathbf{H}_{\tau}(x, y, d+0)$ beneath the buried sheet $(z=d+0)$ as

$$
\begin{aligned}
\mathbf{n} & \times \mathbf{H}_{\tau}(x, y, d+0) \\
& =\int Y\left(x-x^{\prime}, y-y^{\prime} ; \sigma(z)\right) \mathbf{E}_{\tau}\left(x^{\prime}, y^{\prime}, d\right) d x^{\prime} d y^{\prime} .
\end{aligned}
$$

Here $\mathbf{n}=-\mathbf{e}_{z}$ is the upward unit vector; the sign ' $x$ ' denotes the vector product; and, the $2 \times 2$ dyadic tensor $Y(x, y ; \sigma(z))$ has a form (Avdeev et al., 1989)

$$
\begin{aligned}
Y= & -\left(\mathbf{n} \times \nabla_{\tau}\right) \otimes\left(\mathbf{n} \times \nabla_{\tau}\right) \mathscr{B}_{0}\left[\frac{Y^{t}(k, d)}{k}\right] \\
& -\nabla_{\tau} \otimes \nabla_{\tau} \mathscr{B}_{0}\left[\frac{Y^{p}(k, d)}{k}\right],
\end{aligned}
$$




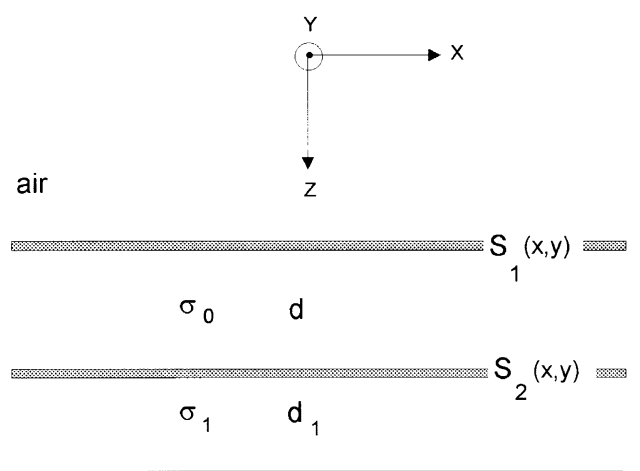

$\sigma_{2} \quad d_{2}$

Fig. 1. An example of a multi-sheet model composed of the surface and buried sheets embedded in layered earth.

where spectral functions $Y^{t}$ and $Y^{p}$ are respectively induction and galvanic admittances of the layered earth beneath the buried sheet; the sign ' $\otimes$ ' stands for the tensor product of vectors, $\mathbf{a} \otimes \mathbf{b}=\left(\begin{array}{ll}a_{x} b_{x} & a_{x} b_{y} \\ a_{y} b_{x} & a_{y} b_{y}\end{array}\right)$; the sign ' $\nabla_{\tau}$ ' denotes horizontal gradient, $\nabla_{\tau}=\left(\partial_{x}, \partial_{y}\right)$; and where the notation $\mathscr{P}_{0}$ denotes the Hankel transform

$$
\mathscr{B}_{0}[f](r)=\frac{1}{2 \pi} \int_{0}^{\infty} f(k) J_{0}(k r) d k .
$$

Here $J_{0}$ is the Bessel function of the first kind and of order zero; $r=\sqrt{x^{2}+y^{2}} ; k=\sqrt{k_{x}^{2}+k_{y}^{2}} ;$ and $k_{x}, k_{y}$ are the spatial wavenumbers.

With $\mathbf{H}_{\tau}(x, y, d+0)$ determined and $\mathbf{H}_{\tau}(x, y, d-0)$, $\mathbf{E}_{\tau}(x, y, d)$ assumed to be known, one can get $S_{2}(x, y)$ from Price's matching condition (Price, 1949)

$$
\begin{gathered}
\mathbf{n} \times \mathbf{H}_{\tau}(x, y, d-0)-\mathbf{n} \times \mathbf{H}_{\tau}(x, y, d+0) \\
=S_{2}(x, y) \mathbf{E}_{\tau}(x, y, d) .
\end{gathered}
$$

Thus, if we know the $\mathbf{E}_{\tau}$ and $\mathbf{H}_{\tau}$ at the roof of the buried sheet, $z=d-0$, we readily obtain the conductance $S_{2}(x, y)$. However the fields are actually assumed known at the surface, $z=0$, rather than at $z=d-0$. So the problem is to continue the fields from the surface down to the roof of the buried sheet.

\subsection{Stable approximation of the downward continued}

\section{EM field}

Our solution of this problem is as follows. The $\mathbf{E}_{\tau}(x, y$, $+0)$ beneath the surface sheet be identical to $\mathbf{E}_{\tau}(x, y, 0)$ at the surface $(z=0)$. At the earth's surface the Price's matching condition can be rewritten as

$$
\begin{aligned}
\mathbf{n} \times \mathbf{H}_{\tau}(x, y,+0)= & \mathbf{n} \times \mathbf{H}_{\tau}(x, y, 0) \\
& -S_{1}(x, y) \mathbf{E}_{\tau}(x, y, 0) .
\end{aligned}
$$

With a knowledge of $S_{1}(x, y)$, this equality gives the magnetic field, $\mathbf{H}_{\tau}(x, y,+0)$, beneath the surface sheet.
Let's next suppose for the moment that $\sigma(z)=\sigma_{0}=$ Const, when $0<z<d$, i.e., the layered earth sandwiched between surface and buried sheets consists of only one layer of conductivity $\sigma_{0}$. Following the Appendix (see transforms (A.13) and (A.14)), the downward continued electric field is

$$
\begin{aligned}
& \mathbf{E}_{\tau}(x, y, d) \\
& \quad=\int Q^{(1)}\left(x-x^{\prime}, y-y^{\prime}\right) \check{\mathbf{E}}_{\tau}\left(x^{\prime}, y^{\prime}, d\right) d x^{\prime} d y^{\prime},
\end{aligned}
$$

where

$$
\begin{aligned}
\check{\mathbf{E}}_{\tau}(x, y, d) & =\mathbf{E}_{\tau}(x, y, 0) \\
& +\int\left(i \omega \mu_{0} Q^{(2)}+\frac{1}{\sigma_{0}} Q^{(3)}\right)\left(x-x^{\prime}, y-y^{\prime}\right) \\
& \cdot \mathbf{H}_{\tau}\left(x^{\prime}, y^{\prime},+0\right) d x^{\prime} d y^{\prime} .
\end{aligned}
$$

Formula (A.15) for kernel $Q^{(1)}$ reflects a dramatic numerical instability of transform (6), since the kernel involves the factor $\cosh (æ d)$ which exponentially increases when $k$ tends to infinity. However sometimes it is very plausible to assume that

$$
æ d \ll 1 .
$$

If this assumption is valid then $\cosh (æ d) \approx 1$, and so, following formula (A.15), $Q^{(1)}\left(x-x^{\prime}, y-y^{\prime}\right) \approx \delta\left(x-x^{\prime}, y-y^{\prime}\right)$, where $\delta$ is Dirac's delta-function. In this case transform (6) degenerates into the identity $\mathbf{E}_{\tau}=\check{\mathbf{E}}_{\tau}$ and thus one can choose field $\check{\mathbf{E}}_{\tau}(x, y, d)$, determined by transform (7), as the desired stable approximation of the downward continued electric field. In a similar way transform (A.18) defines the stable approximation of the downward continued magnetic field

$$
\begin{aligned}
& \check{\mathbf{H}}_{\tau}(x, y, d-0) \\
& =\mathbf{H}_{\tau}(x, y,+0) \\
& \quad+\int\left(\sigma_{0} Q^{(2)}+\frac{1}{i \omega \mu_{0}} Q^{(3)}\right)\left(x-x^{\prime}, y-y^{\prime}\right) \\
& \quad \quad \mathbf{E}_{\tau}\left(x^{\prime}, y^{\prime}, 0\right) d x^{\prime} d y^{\prime} .
\end{aligned}
$$

It should be noted that in practice the assumption (8) may be considered as valid when

$$
d \ll L, \quad d \ll C .
$$

Here $C=\sqrt{\frac{2}{\omega \mu_{0} \sigma_{0}}}$ is a skin depth, and $L$ is the smallest horizontal size of the surface and buried inhomogeneities.

Now, in the general case, when the earth between surface and buried sheets consists of a stack of more than one homogeneous layer, transforms (7) and (9) are to be recursively applied for each layer of the stack.

\section{Imaging of a Buried Sheet Conductance}

Summing up, the overall inversion scheme involves the following three steps.

Step 1. By Prices' condition (5), we derive the horizontal magnetic field beneath the surface sheet, $z=+0$.

Step 2. By transforms (7) and (9), we continue the horizontal electric and magnetic fields to the roof of the buried sheet, $z=d-0$. 

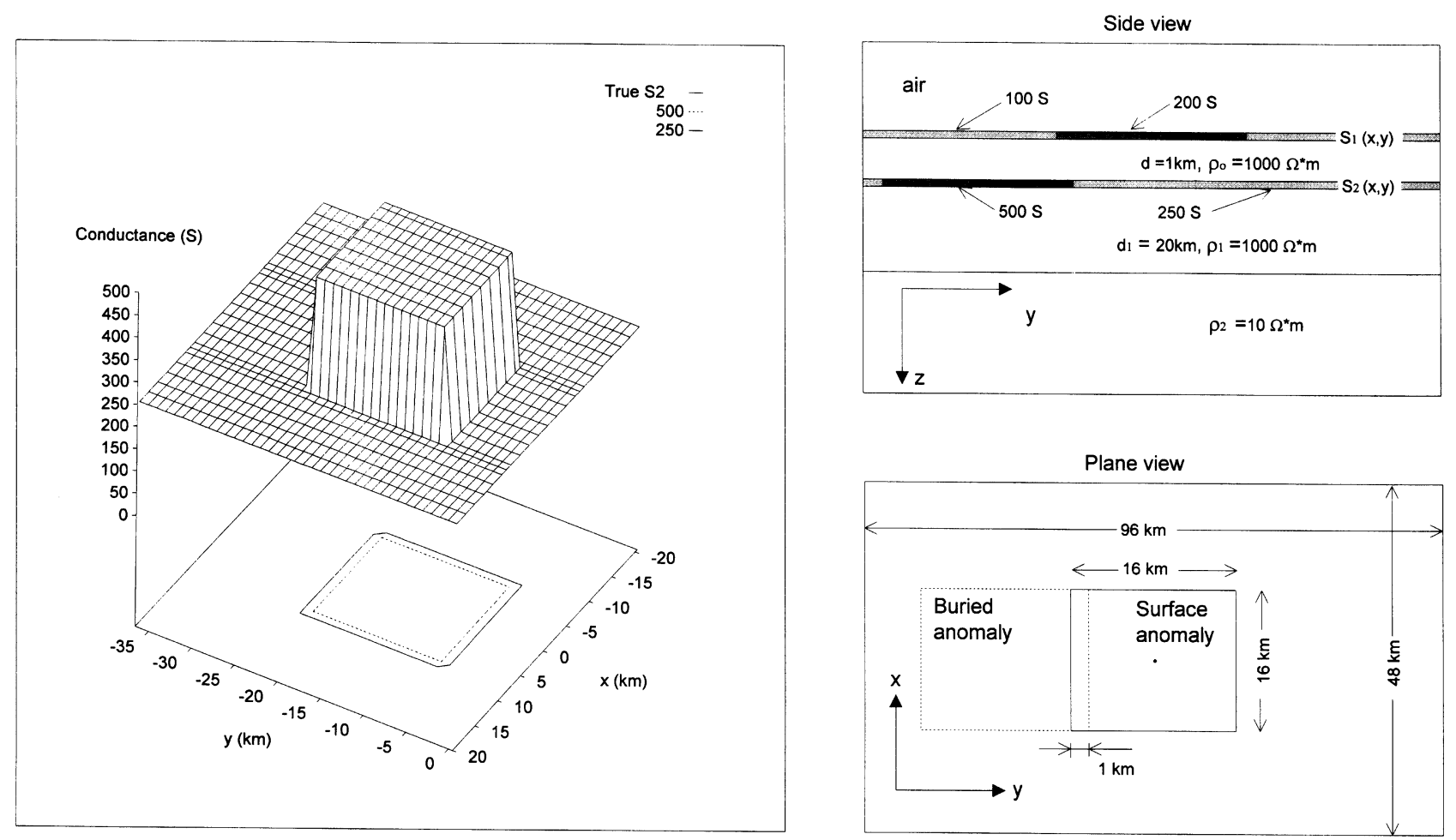

Fig. 2. The buried sheet conductance (left panel) of the multi-sheet model (right panels) used for imaging.

Step 3. By transform (1), we derive the horizontal magnetic field beneath the buried sheet, $z=d+0$, and, at last, determine $S_{2}$ of equation (4) as

$$
\begin{aligned}
S_{2}(x, y) & \left|\mathbf{E}_{\tau}(x, y, d)\right|^{2} \\
& \cdot\left(\mathbf{n} \times \mathbf{H}_{\tau}(x, y, d-0)-\mathbf{n} \times \mathbf{H}_{\tau}(x, y, d+0)\right) \\
& \cdot \mathbf{E}_{\tau}^{*}(x, y, d) .
\end{aligned}
$$

Here superscript ' $*$ ' means complex conjugation. We take the in-phase part of the right side of equality (11) as a desired $S_{2}(x, y)$, and use its out-of-phase part as a measure of consistency of our multi-sheet imaging. To perform convolutions over the $(x, y)$-plane in (1), (7) and (9) we use the numerical scheme described in (Avdeev et al., 1997).

To perform Hankel transforms of type (3) we use the FHT code of Christensen (1990) which is based on the technique of Johansen and Sorensen (1979).

\section{Numerical Examples}

We constructed a multi-sheet model to test our inversion scheme. It consists of a three-layered earth with surface and buried sheets (Fig. 2). The surface sheet contains a $16 \times$ $16 \mathrm{~km}^{2}$ anomaly of conductance $200 \mathrm{~S}$. Everywhere out of the anomaly the surface sheet conductance is $100 \mathrm{~S}$. The buried sheet is placed at depth of $1 \mathrm{~km}$ and also contains a $16 \times 16 \mathrm{~km}^{2}$ anomaly of conductance $500 \mathrm{~S}$. This anomaly is $15 \mathrm{~km}$ shifted along $y$-axis with respect to the surface anomaly. Everywhere out of the anomaly, the buried sheet conductance is $250 \mathrm{~S}$. The geoelectric parameters of the three-layered earth are as follows: the top layer conductivity $\sigma_{0}=10^{-3} \mathrm{~S} / \mathrm{m}$, and thickness $d=1 \mathrm{~km}$, the middle layer with $\sigma_{1}=10^{-3} \mathrm{~S} / \mathrm{m}$ and $d_{1}=20 \mathrm{~km}$, and the lower half space with $\sigma_{2}=10^{-1} \mathrm{~S} / \mathrm{m}$.

We adopted a numerical mesh of $N_{x} \times N_{y}=64 \times 96$ cells for each sheet, with a cell size of $1 \times 1 \mathrm{~km}^{2}$ and with the centre $(x=0, y=0)$ coinciding with the centre of the surface anomaly. Two kinds of excitations of $10 \mathrm{~s}$ period were considered: a $y$-polarized plane wave and a $y$-directed electric dipole grounded at the centre of the surface anomaly. We calculated the horizontal electric and magnetic fields at the earth's surface using a 3-D integral equation solution (Avdeev et al., 1997). Then we applied our inversion scheme to those data. Our numerical experiments discovered that for the case of dipole excitation it is better to use the scattered EM field rather than the total field. Here scattered field means a difference between total and background fields, where the background one is a field for the same model but without a buried sheet. Clearly, the background field can be readily calculated, since $\sigma(z)$ and $S_{1}(x, y)$ are assumed to be known. Figures 3 and 4 demonstrate the images obtained for the plane wave and for the dipole excitations. It is seen that for both excitations the in-phase parts of the images quite correctly reproduce the location, shape and conductance of the buried anomaly. Maximal in-phase conductances, $502 \mathrm{~S}$ for plane wave excitation and $505 \mathrm{~S}$ for dipole excitation, are very close to the true conductance, $500 \mathrm{~S}$, of the buried anomaly. Out-of-phase conductances are small, not exceeding a few percent of the in-phase conductance. This result indicates the reliability of the multi-sheet inversion. 

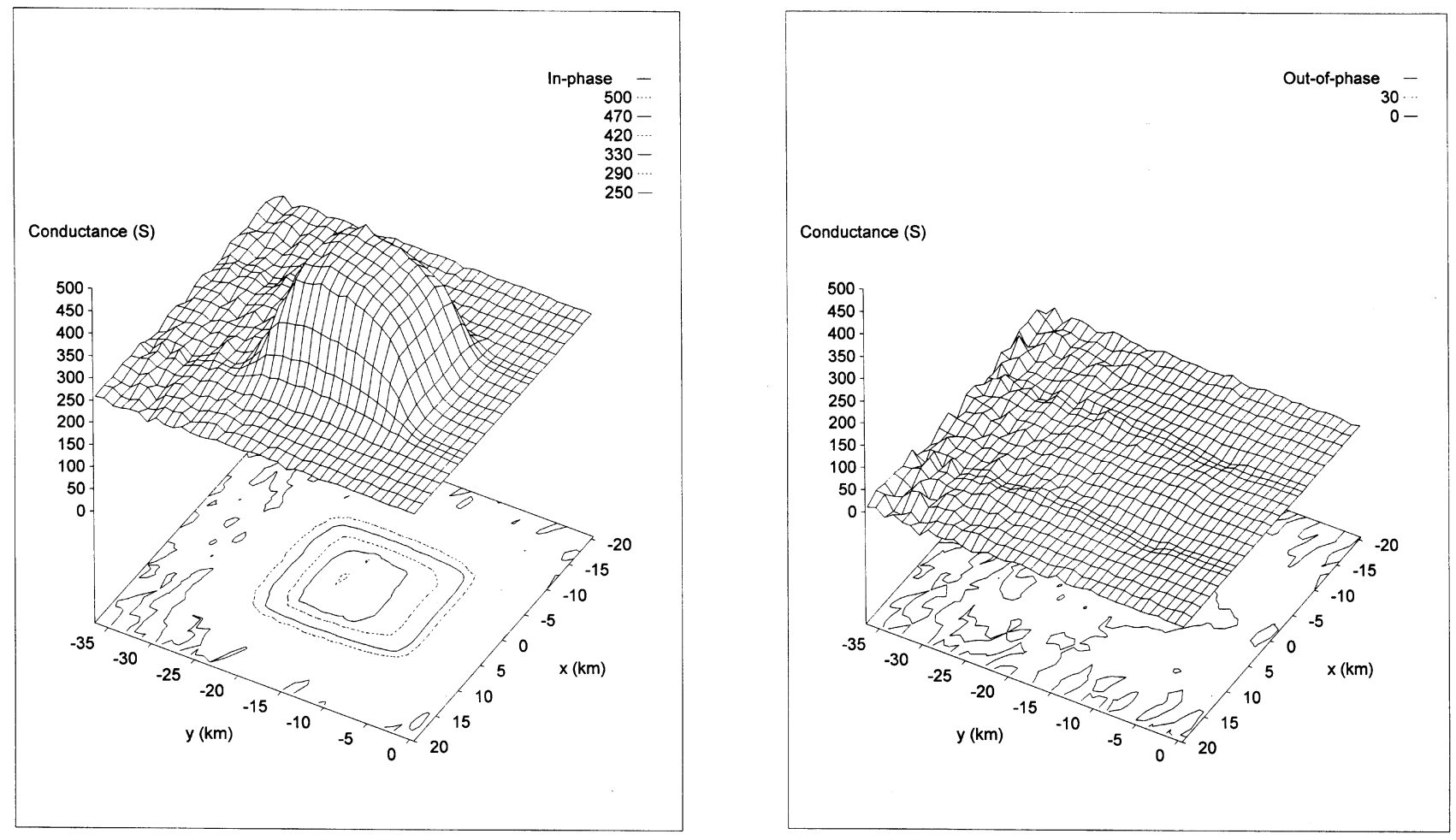

Fig. 3. The $0.1 \mathrm{~Hz}$ MT image of the model in Fig. 1. The left panel shows the in-phase part of the buried sheet conductance $S_{2}$, while the right panel presents the out-of-phase part.
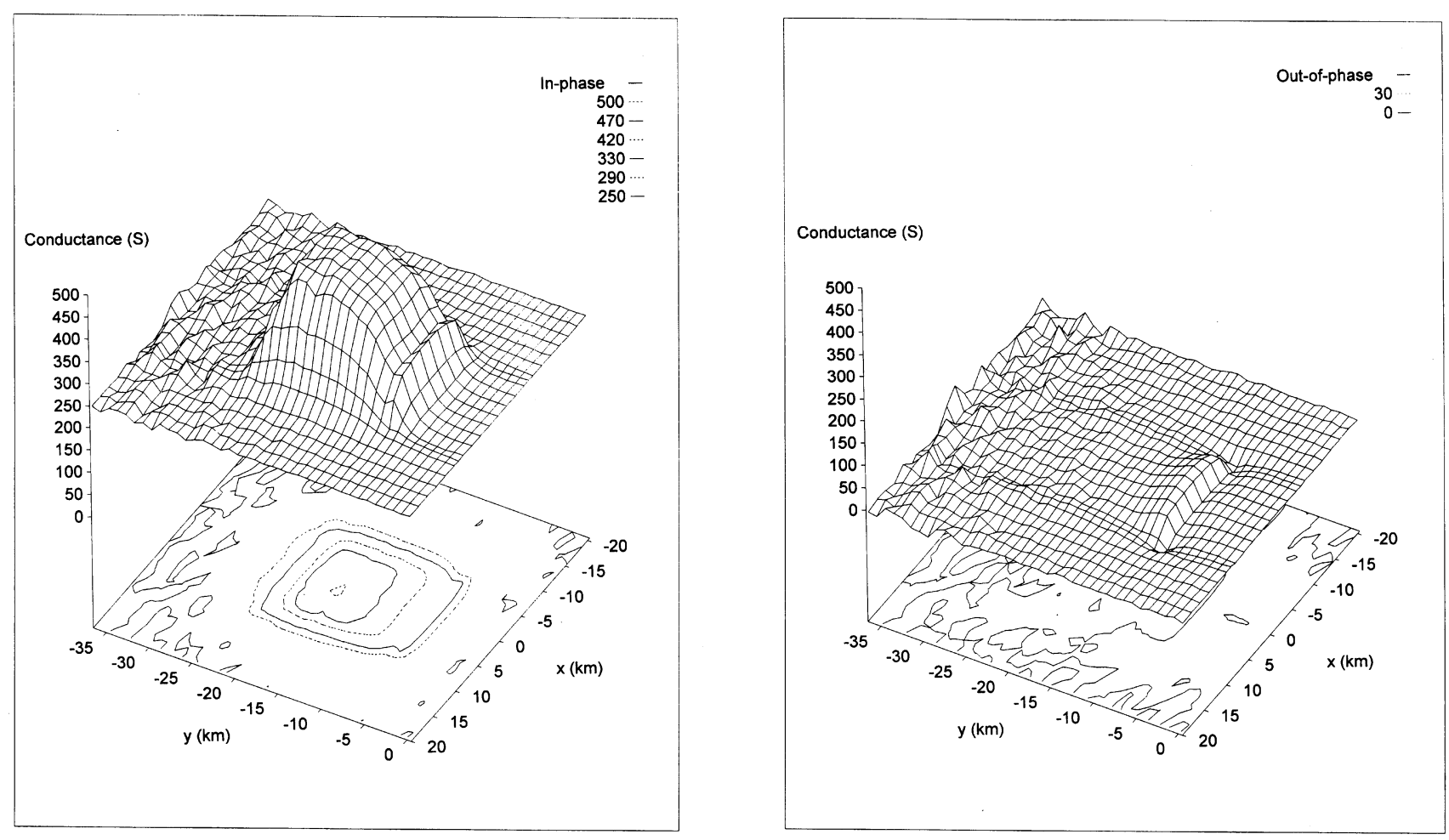

Fig. 4. The $0.1 \mathrm{~Hz}$ HED image of the model in Fig. 1. The left panel shows the in-phase part of the buried sheet conductance $S_{2}$, while the right panel presents the out-of-phase part. 


\section{Conclusion}

In this paper we have proposed a stable and fast inversion scheme of imaging underground structures. As an example, this scheme takes $12 \mathrm{~s}$ on a Pentium-100 MHz per frequency and per excitation to invert $64 \times 96$ array of EM data. The reason for its speed is that the inversion doesn't need forward modelling. However this merit appears to be counterbalanced by the fact that a spatial coverage and simultaneity of EM measurements are required. We believe that our inversion scheme may have various useful geophysical applications, such as the detection of crustal anomalies, EM monitoring of seismotectonic processes and of hydrocarbon reservoir changes, and mapping of pollution zones.

Acknowledgments. We thank Boris Singer for many valuable discussions of a multi-sheet inversion given to authors during his stay at the EM Modelling Laboratory of IZMIRAN. We are also grateful to anonymous referees for their useful suggestions and comments on the manuscript and to William Frangos for his corrections of the English. The research described in this publication was made possible in part by grant RIA000 from the International Science Foundation and grants no. 96-05-66006 and no.97-05-65725 from the Russian Foundation for Basic Research.

\section{Appendix. EM Field Downward Continuation Across a Homogeneous Layer}

In this Appendix we derive an explicit form for the horizontal EM field continuation from the earth's surface, $z=+0$, down to a depth, $z=d-0$. Maxwell's equations within the homogeneous layer $(0<z<d)$ of conductivity $\sigma_{0}$ read

$$
\begin{aligned}
& \nabla \times \mathbf{H}=\sigma_{0} \mathbf{E}, \\
& \nabla \times \mathbf{E}=i \omega \mu_{0} \mathbf{H} .
\end{aligned}
$$

Let's perform the Fourier transform

$$
\mathbf{E}_{\tau}\left(k_{x}, k_{y}, z\right)=\int \mathbf{E}_{\tau}(x, y, z) \mathrm{e}^{-i x k_{x}-i y k_{y}} d x d y,
$$

of horizontal electric field $\mathbf{E}_{\tau}$ into $\left(k_{x}, k_{y}\right)$-domain. In this domain we decompose $\mathbf{E}_{\tau}(\mathbf{k}, z)$ as

$$
\mathbf{E}_{\tau}=-\mathscr{C}^{t} \frac{\mathbf{n} \times i \mathbf{k}}{k}-\mathscr{E}^{p} \frac{i \mathbf{k}}{k}
$$

via scalar potentials $\mathscr{E}^{t}(\mathbf{k}, z)$ and $\mathscr{E}^{p}(\mathbf{k}, z)$. Here $\mathbf{k}=$ $\left(k_{x}, k_{y}\right)$, and $k=|\mathbf{k}|$. Similarly we decompose horizontal magnetic field $\mathbf{H}_{\tau}(\mathbf{k}, z)$ as

$$
\mathbf{n} \times \mathbf{H}_{\tau}=-\mathscr{H}^{t} \frac{\mathbf{n} \times i \mathbf{k}}{k}-\mathscr{H}^{p} \frac{i \mathbf{k}}{k} .
$$

Substituting decompositions (A.3) and (A.4) into Maxwell's equations (A.1) transformed beforehand into $\left(k_{x}, k_{y}\right)$ domain, and gathering terms involving vectors $\frac{\mathbf{n}_{\times i} \mathbf{k}}{k}$ and $\frac{i \mathbf{k}}{k}$ we derive the system

$$
\left\{\begin{array}{l}
\partial_{z} \mathscr{E}^{t}=i \omega \mu_{0} \mathscr{H}^{t} \\
\partial_{z} \mathscr{H}^{t}=\frac{\mathfrak{x}^{2}}{i \omega \mu_{0}} \mathscr{E}^{t}
\end{array}\right.
$$

with respect to $\mathscr{E}^{t}, \mathscr{H}^{t}$, and the system

$$
\left\{\begin{array}{l}
\partial_{z} \mathscr{E}^{p}=-\frac{\mathfrak{x}^{2}}{\sigma_{0}} \mathscr{H}^{p} \\
\partial_{z} \mathscr{H}^{p}=-\sigma_{0} \mathscr{E}^{p}
\end{array}\right.
$$

with respect to $\mathscr{E}^{p}, \mathscr{H}^{p}$. Here $\mathfrak{x}^{2}=k^{2}-i \omega \mu_{0} \sigma_{0}$. System (A.5) is equivalent to the second order differential equation

$$
\partial_{z}^{2 \mathscr{E} t}=\mathfrak{x}^{2 \mathscr{E} t}
$$

The solution of this equation at depth $z=d-0$ can be written via the solution at depth $z=+0$ as

$$
\begin{aligned}
\mathscr{E}^{t}(\mathbf{k}, d-0)= & \cosh (æ d) \mathscr{E}^{t}(\mathbf{k},+0) \\
& +\frac{1}{\mathfrak{x}} \sinh (\mathfrak{x} d) \partial_{z} \mathscr{E}^{t}(\mathbf{k},+0) .
\end{aligned}
$$

From (A.5) we have $\partial_{z} \mathscr{E}^{t}(\mathbf{k},+0)=i \omega \mu_{0} \mathscr{H}^{t}(\mathbf{k},+0)$, and thus equation (A.8) reads

$$
\begin{aligned}
& \mathscr{E}^{t}(\mathbf{k}, d-0) \\
& =\cosh (æ d)\left(\mathscr{E}^{t}(\mathbf{k},+0)+\frac{i \omega \mu_{0}}{\mathfrak{x}} \tanh (æ d) \mathscr{H}^{t}(\mathbf{k},+0)\right) .
\end{aligned}
$$

In a similar way we derive

$$
\begin{aligned}
& \mathscr{E}^{p}(\mathbf{k}, d-0) \\
& \quad=\cosh (æ d)\left(\mathscr{E}^{p}(\mathbf{k},+0)-\frac{æ}{\sigma_{0}} \tanh (æ d) \mathscr{H}^{p}(\mathbf{k},+0)\right) .
\end{aligned}
$$

From decompositions (A.3) and (A.4) it follows that

$$
\begin{gathered}
\mathscr{E}^{t}=\frac{\mathbf{n} \times i \mathbf{k}}{k} \cdot \mathbf{E}_{\tau}, \mathscr{H}^{t}=\frac{\mathbf{n} \times i \mathbf{k}}{k} \cdot \mathbf{n} \times \mathbf{H}_{\tau}, \\
\mathscr{E}^{p}=\frac{i \mathbf{k}}{k} \cdot \mathbf{E}_{\tau}, \quad \mathscr{H}^{p}=\frac{i \mathbf{k}}{k} \cdot \mathbf{n} \times \mathbf{H}_{\tau} .
\end{gathered}
$$

Substitutions of (A.11) into (A.9), (A.10) and consequent substitutions of the results into (A.3) give

$$
\begin{aligned}
& \mathbf{E}_{\tau}(\mathbf{k}, d-0)=\cosh (æ d) \\
& \cdot\left(\mathbf{E}_{\tau}(\mathbf{k},+0)\right. \\
& \quad-i \omega \mu_{0} \frac{\tanh (æ d)}{æ}\left(\frac{\mathbf{n} \times i \mathbf{k}}{k}\right) \otimes \frac{i \mathbf{k}}{k} \mathbf{H}_{\tau}(\mathbf{k},+0) \\
& \left.\quad-\frac{1}{\sigma_{0}} \mathfrak{t a n h}(æ d) \frac{i \mathbf{k}}{k} \otimes\left(\frac{\mathbf{n} \times i \mathbf{k}}{k}\right) \mathbf{H}_{\tau}(\mathbf{k},+0)\right) .
\end{aligned}
$$

Ultimately, transforming (A.12) into the $(x, y)$-domain, we obtain the downward continued electric field

$$
\begin{aligned}
\mathbf{E}_{\tau}(x, y, d-0 & =\int Q^{(1)}\left(x-x^{\prime}, y-y^{\prime}\right) \\
& \check{\mathbf{E}}_{\tau}\left(x^{\prime}, y^{\prime}, d-0\right) d x^{\prime} d y^{\prime},
\end{aligned}
$$

where

$$
\begin{aligned}
& \check{\mathbf{E}}_{\tau}(x, y, d-0) \\
& \quad=\mathbf{E}_{\tau}(x, y,+0) \\
& \quad+\int\left(i \omega \mu_{0} Q^{(2)}+\frac{1}{\sigma_{0}} Q^{(3)}\right)\left(x-x^{\prime}, y-y^{\prime}\right) \\
& \quad+\mathbf{H}_{\tau}\left(x^{\prime}, y^{\prime},+0\right) d x^{\prime} d y^{\prime}
\end{aligned}
$$

and where

$$
Q^{(1)}=\mathscr{B}_{0}[k \cosh (æ d)],
$$




$$
\begin{aligned}
& Q^{(2)}=-\left(\mathbf{n} \times \nabla_{\tau}\right) \otimes \nabla_{\tau} \mathscr{B}_{0}\left[\frac{\tanh (æ d)}{k æ}\right], \\
& Q^{(3)}=-\nabla_{\tau} \otimes\left(\mathbf{n} \times \nabla_{\tau}\right) \mathscr{B}_{0}\left[\frac{æ \tanh (æ d)}{k}\right] .
\end{aligned}
$$

Definitions of tensor product $\otimes$ and of Hankel transform $\mathscr{B}_{0}$ are presented in Subsection 3.1.

In a similar way we derive the downward continued magnetic field

$$
\begin{aligned}
\mathbf{H}_{\tau}(x, y, d-0 \neq & \int Q^{(1)}\left(x-x^{\prime}, y-y^{\prime}\right) \\
& \check{\mathbf{H}}_{\tau}\left(x^{\prime}, y^{\prime}, d-0\right) d x^{\prime} d y^{\prime},
\end{aligned}
$$

where

$$
\begin{aligned}
& \check{\mathbf{H}}_{\tau}(x, y, d-0) \\
& =\mathbf{H}_{\tau}(x, y,+0) \\
& \quad+\int\left(\sigma_{0} Q^{(2)}+\frac{1}{i \omega \mu_{0}} Q^{(3)}\right)\left(x-x^{\prime}, y-y^{\prime}\right) \\
& \quad \cdot \mathbf{E}_{\tau}\left(x^{\prime}, y^{\prime}, 0\right) d x^{\prime} d y^{\prime} .
\end{aligned}
$$

\section{References}

Alumbaugh, D. L., Iterative electromagnetic Born inversion applied to earth conductivity imaging, Ph.D. Thesis, Univ. Calif., Berkeley, 1993.

Avdeev, D. B., B. Sh. Singer, and E. B. Fainberg, The inverse problem of geoelectrics for thin sheet models, in Fundamental Problems of Marine Explorations, edited by M. S. Zhdanov, pp. 24-47, IZMIRAN, Moscow, 1986 (in Russian).
Avdeev, D. B., E. B. Fainberg, and B. Sh. Singer, On applicability of Tikhonov-Cagniard magnetotelluric model for sounding of a non-uniform medium, Phys. Earth Planet. Int., 53, 343-349, 1989.

Avdeev, D. B., V. G. Dubrovsky, E. B. Fainberg, O. V. Pankratov, and B. Sh. Zinger, Deep electromagnetic sounding in Turkmenia, Geophys. J. Int., 118, 467-484, 1994.

Avdeev, D. B., A. V. Kuvshinov, O. V. Pankratov, and G. A. Newman, High-performance three-dimensional electromagnetic modelling using modified Neumann series. Wide-band numerical solution and examples, J. Geomag. Geoelectr., 49, 1519-1539, 1997.

Christensen, N. B., Optimized Fast Hankel Transform filters, Geophys Prospect., 38, 545-568, 1990.

Eaton, P. A., Three-dimensional electromagnetic inversion using integral equations, Geophys. Prospect., 37, 407-426, 1989.

Johansen, H. K. and K. Sorensen, Fast Hankel Transforms, Geophys. Prospect., 27, 876-901, 1979.

Lee, K. H. and G. O. Xie, A new approach to imaging with low-frequency electromagnetic fields, Geophys., 58, 780-796, 1993.

Madden, T. R. and R. L. Mackie, Three-dimensional magnetotelluric modeling and inversion, Proc. IEEE, 77, 318-333, 1989.

Newman, G. A., Crosswell electromagnetic inversion using integral and differential equations, Geophys., 60, 899-911, 1995.

Oldenburg, D. W. and R. G. Ellis, Inversion geophysical data using an approximate inverse mapping, Geophys. J. Int., 105, 325-351, 1991.

Price, A. T., The induction of electric currents in nonuniform sheets and shells, Quart. J. Mech. Appl. Math., 2, 283-310, 1949.

Singer, B. Sh. and E. B. Fainberg, Electromagnetic Induction in Inhomogeneous Thin Sheets, 234 pp., IZMIRAN, Moscow, 1985 (in Russian).

D. B. Avdeev (e-mail: avdeev@gemri.msk.ru), A. V. Kuvshinov, and O. V. Pankratov 